\title{
Expresión Morfológica de la Arteria Basilar. Un Estudio con Material de Autopsia de Individuos Colombianos
}

\author{
Morphological Expression of the Basilar Artery. \\ A Study with Colombian Population's Autopsy Material
}

Forero Pedro Luis*; Ballesteros Luis Ernesto** \& Ramírez Luis Miguel ${ }^{* * *}$

\begin{abstract}
FORERO, P. L.; BALLESTEROS, L. E \& RAMÍREZ, L. M. Expresión morfológica de la arteria basilar. Un estudio con material de autopsia de individuos colombianos. Int. J. Morphol., 31(3):1090-1096, 2013.

RESUMEN: La arteria basilar $(\mathrm{AB})$ presenta una expresión variable, la cual es determinante en la etiología de eventos clínicos que comprometen el tallo cerebral. El propósito de este trabajo fue determinar la expresión morfológica de la AB en una muestra de población colombiana. Fueron estudiados 100 tallos cerebrales de individuos a quienes se les practicó autopsia en el Institutode Medicina Legal de Bucaramanga. El sistema vertebro-basilar fue perfundido con resina sintética y evaluado los calibres, trayectorias, niveles de origen y finalización de la $\mathrm{AB}$. La AB presentó trayectoria rectilínea en $68 \%$, desviación a la derecha en $12 \%$, sinuosa en $10 \%$ y desviación a la izquierda en 10\%. La longitud total de la AB fue 30,2 mm DE 4,07 y la longitud con relación al origen de la arteria Cerebelar superior fue $28,1 \mathrm{~mm}$ DE 3,84. Los calibres proximal y distal de la AB fueron 3,96 mm DE 0,48 mm y 3,7 mm DE 0,58 respectivamente. Con relación a las arterias de origen de la $\mathrm{AB}$, se observó hipoplasia ( $\geq 2 \mathrm{~mm})$ de la arteria vertebral derecha en el $8 \%$ y de la arteria vertebral izquierda en el $11 \%(\mathrm{P}=0,30)$. Se observó el origen de la $\mathrm{AB}$ a tres niveles del surco pontomedular: a nivel en el $43 \%$, por arriba de este en el $30 \%$ y por debajo de este surco en $27 \%$ de los casos. Las trayectorias sinuosas y desviadas de la AB observadas y la presencia de hipoplasias de la arteria vertebral son ligeramente superiores a los señalados en estudios previos, mientras que su longitud se ubica en un rango medio y su calibre es ligeramente inferior a lo señalado en la literatura
\end{abstract}

PALABRAS CLAVE: Variación anatómica; Arteria basilar; arteria vertebral; tallo cerebral.

\section{INTRODUCCIÓN}

La arteria basilar $(\mathrm{AB})$ se forma usualmente al nivel del surco bulbomedulary de la unión de los segmentos intracraneanos de las arterias vertebrales izquierda y derecha. Cursa sobre la superficie anterior del puente y se bifurca dando lugar a las arterias cerebrales posteriores al nivel de la fosa interpeduncular (Afifi \& Bergman 2008; Moore et al., 2010; Drake et al., 2010). Ocasionalmente, la AB continúa al nivel del puente como dos troncos (expresión fenestrada) que la recorren longitudinalmente, los cuales luego de un trayecto variable se anastomosan para formar una estructura única (Yoshimoto et al., 1992; Goldstein et al., 1999; Dimmick \& Faulder, 2009; Dodevski et al., 2011; Uchino et al., 2012).

La AB presenta evidentes variaciones en su expresión morfológica relacionadas con su origen, longitud, trayectoria y calibre (Torche et al., 1992; Akar et al., 1994; SchulteAltedorneburg et al., 2000; Mandiola et al., 2004; Pai et al.,
2007; Songur et al., 2008). Además del surco pontomedular, esta puede originarse por debajo o por arriba de este surco referente (Stopford, 1916; Wojtowicz et al., 1989;Santos-Franco et al., 2006; Stopforf; Songur et al.).

La trayectoria de la AB sobre el surco del puente, puede ser rectilínea (45-80\%), desviada hacia la derecha o izquierda y en escaso porcentaje presenta una forma zigzagueante (Akimoto, H, 1979; Schulte-Altedorneburg et al.; Pai et al.; Torche et al.; Wojtowicz et al.). En la mayoría de los casos (60\%) finaliza a nivel del surco superior del puente y en menor frecuencia por arriba o por debajo del mencionado surco (Smoker et al., 1986; Pai et al.). Ligeras diferencias en relación al diámetro $(3,8-5 \mathrm{~mm})$ y longitud (28-33mm) de la $\mathrm{AB}$ han sido reportados por diversos trabajos (Bush, W. 1966; Wollschlaeger et al., 1967; Songur et al.; Smoker et al.; Akimoto; Pai et al., Santos-Franco et al.,; Er et al., 2008; Hong et al., 2009).

* MD, Esp Patol. Profesor asistente Universidad Industrial de Santander, Colombia. Instituto de Medicina Legal y Ciencias Forenses
** MD, MSc. Profesor titular Universidad Industrial de Santander, Colombia

**** DDS, MSc. Profesor asociado Universidad de Antioquía, Colombia. 
La identificación de variaciones en la $\mathrm{AB}$ puede ser útil en la prevención de complicaciones durante el tratamiento endovascular y en el diagnóstico de aneurismas asociados. Las variaciones de la $\mathrm{AB}$ no determinan per se, diferencias en el suministro de sangre en los territorios irrigados, pero las variaciones sumadas a otros factores como estenosis, ateromatosis, embolia o trombosis son causa de cuadros clínicos originados en el tallo cerebral (Wollschlaeger $e t$ al., 1967; Schulte-Altedorneburg et al.; Pico et al., 2006; Deng et al., 2012). Las expresiones morfológicas de la $\mathrm{AB}$ han sido descritas mediante técnicas de disección clásica, inyección de sus lechos vasculares o estudios imagenológicos en otros grupos poblacionales (Stopford; Smoker et al.,; Mandiola et al., 1995; Pai et al.; Santos-Franco et al.; Wojtowicz et al.). Con el propósito de obtener información propia de referencia, este estudio contó con muestras cadavéricas frescas de sujetos colombianos.

\section{MATERIAL Y MÉTODO}

Se evaluó las AB obtenidas de 100 especímenes cadavéricos frescos a quienes se les Practicó necropsia en el Instituto de Medicina Legal de Bucaramanga (Colombia). La muestra cumplió con los siguientes criterios de inclusión: sujetos masculinos, con edades entre 18-75 años, de ancestria mestiza, sin evidencia de haber fallecido por traumatismo directo o patologías que comprometieran al sistema nervioso central.

Cada especimen fue sometido a canalización bilateral de los segmentos proximales de las arterias vertebrales. A través de éstas, se practicó lavado y prefijado del encéfalo con formaldehido al $3 \%$ y posterior inyección de $100 \mathrm{cc}$ de resina semisintética (mezcla de palatal E210® Basf $80 \mathrm{cc}$; estireno $20 \mathrm{cc}$ ) impregnada de color rojo mineral. Una vez obtenida la polimerización de la resina (30 minutos), se practicó la exéresis del encéfalo. Posteriormente, la muestra se sometió a fijación con formaldehido al $10 \%$ durante 10 días.

Una vez lograda una adecuada fijación de la masa encefálica, se practicó resección de los bloques de tallos cerebrales y cerebelos y con equipo de micro disección se liberó las leptomeninges, maniobra que permitió identificar plenamente cada una de las estructuras constituyentes del sistema vertebro basilar. Posteriormente, se registraron las diversas expresiones morfológicas de la $\mathrm{AB}$ relacionadas con su nivel de origen, tomando como referente el surco pontomedular; longitud, calibre y trayectoria (rectilínea, desviada a derecha o izquierda y sinuosa). Igualmente, se determinó el nivel de finalización de la $\mathrm{AB}$ con referencia al surco superior del puente. Se midió el diámetro externo de la $\mathrm{AB}$ al nivel de su origen y en su finalización. Para cada una de las evaluaciones morfo métricas se utilizó calibrador digital (Mitotuyo ${ }^{\circledR}$ ).

De cada especimen estudiado se tomaron fotografías digitales (Cámara Canón T2i) que sustentan las observaciones registradas. Los datos obtenidos se digitaron en Excel y los análisis estadísticos se realizaron en STATA 8.0. Las variables continuas se describieron con sus promedios y dispersiones, las variables nominales con sus proporciones. Se realizaron las pruebas estadísticas de chi cuadrado $\left(\mathrm{X}^{2}\right)$ y test de Student $(t)$ aceptando un error alfa hasta de un 5\%.

\section{RESULTADOS}

Se evaluaron 100 segmentos de $\mathrm{AB}$ obtenidos de especímenes cadavéricos masculinos con edad promedio de 33,8 años DE 16,3. Se observó el origen de la $A B$ al nivel del surco pontomedular en 43 especímenes (43\%); por arriba de este surco y a una distancia de $4 \mathrm{~mm}$ DE 1,30 en el $30 \%$ de las piezas evaluadas (Figs. 1,2). Igualmente, en el $27 \%$ de los casos el origen de la AB fue distal al surco pontomedular, a una distancia promedio de esta estructura de referencia de 3,26 mm DE 1,44 (Fig. 3). La diferencia de la distancia de origen proximal de la $\mathrm{AB}$ al surco pontomedular con relación al origen distal no es estatistamente significativa $(\mathrm{P}=0,35)$. El origen de la $\mathrm{AB}$ a nivel del surco pontomedular es significativamente menor $(\mathrm{P}=0,001)$ con relación al número de casos originados proximal y distalmente al surco de referencia.

La $\mathrm{AB}$ presentó trayectoria rectilínea en el $68 \%$ de las muestras, cursando de manera longitudinal sobre el surco basilar del puente, cifra que aumentó al $80 \%$ cuando se evaluaron solamente los especímenes de menores de 40 años (Fig. 1); se observó desviada a la derecha en el 12\% (Fig. 2), sinuosa en 10 (Fig.4) y desviada a la izquierda en el 10\% de los especímenes (Fig.5).

Con relación a las arterias que dan origen a la $\mathrm{AB}$, se observó hipoplasia ( $\geq 2 \mathrm{~mm}$ ) de la arteria vertebral derecha en el $8 \%$ y de la arteria vertebral izquierda en el $11 \%$, sin que esta diferencia de lado fuera significativa $(P=0,30)$, (Fig.6). La hipoplasia de las arterias vertebrales izquierdas fue más acentuado (1,75 mm DE 0,24 AVI; $1,91 \mathrm{~mm} \mathrm{DE}$ 0,10 AVD).

La longitud total de la $\mathrm{AB}$ fue $30,2 \mathrm{~mm} \mathrm{DE} 4,07$, mientras que la distancia desde su origen a la emergencia de la arteria cerebelar superior fue de $28,1 \mathrm{~mm}$ DE 3,84. Los 
calibres proximal y distal de la $\mathrm{AB}$ fueron $3,96 \mathrm{~mm} \mathrm{DE} 0,48$ $\mathrm{mm}$ y $3,7 \mathrm{~mm}$ DE 0,58 respectivamente, presentando una reducción de su calibre al final de su curso de $6,6 \%$.

La AB finalizó dando origen a las arterias cerebrales posteriores al nivel del surco superior del puente en el $55 \%$ (Fig.7); distal al surco en $29 \%$, a una distancia de 2,94 $\mathrm{mm}$ DE 1,26 y proximal en $16 \%$, a una distancia del surco referente de 2,19 mm DE 0,57. En todos los casos el nervio oculomotor emergió desde la fosa interpeduncular delimitado por el segmento precomunicante de la arteria cerebral posterior y la arteria cerebelar superior. En la muestra evaluada la $\mathrm{AB}$ no presentó expresiones fenestradas.

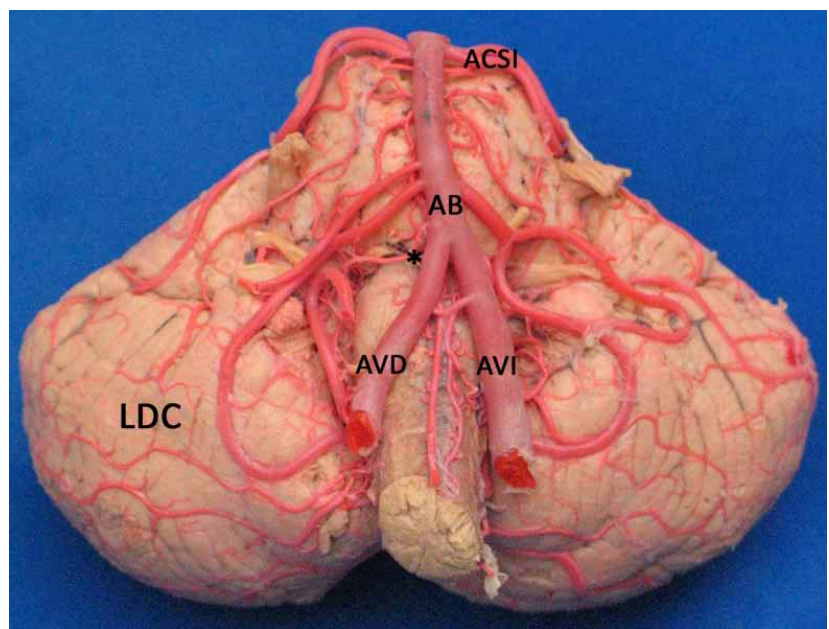

Fig. 1. Sistema arterial vertebrobasilar. Origen de arteria basilar al nivel del surco bulbomedular y de trayectoria rectilínea AB. Arteria basilar; LCD. Lóbulo derecho del cerebelo; AVD. Arteria vertebral derecha; AVI. Arteria vertebral izquierda; ACSI. Arteria cerebelar superior izquierda; Asterisco. Surco pontomedular.

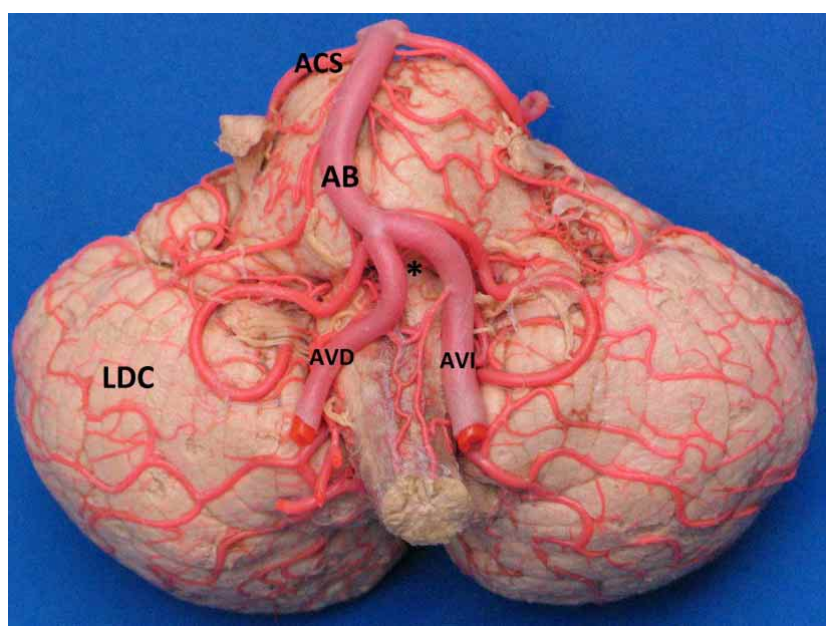

Fig. 2. Sistema arterial vertebrobasilar. Origen de arteria basilar por arriba del surco bulbopontino y desviada a la derecha. AB. Arteria basilar; LCD. Lóbulo derecho del cerebelo; AVD. Arteria vertebral derecha; AVI. Arteria vertebral izquierda; ACS. Arteria cerebelar superior; Asterisco. Surco pontomedular.

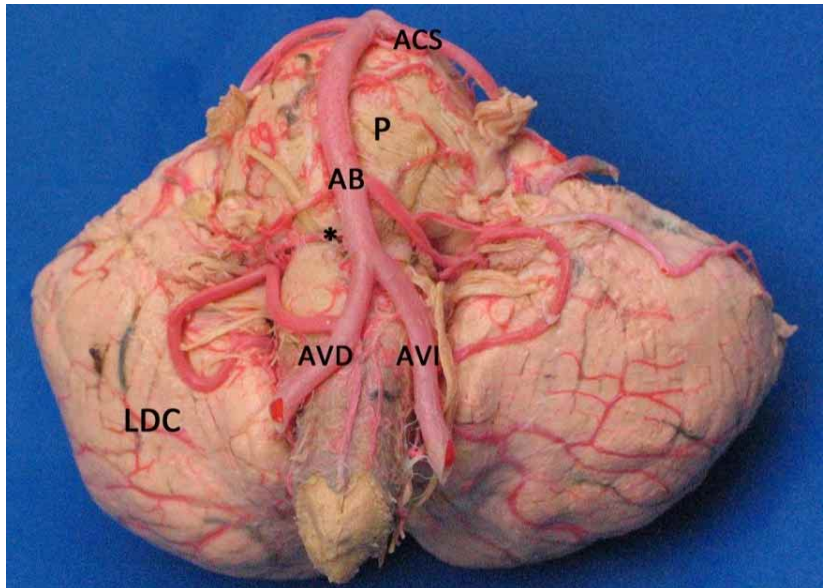

Fig. 3. Sistema arterial vertebrobasilar. Origen de arteria basilar por debajo del surco bulbopontino. AB. Arteria basilar; LCD. Lóbulo derecho del cerebelo; AVD. Arteria vertebral derecha; AVI. Arteria vertebral izquierda; ACS. Arteria cerebelar superior; P. Puente; Asterisco. Surco pontomedular.

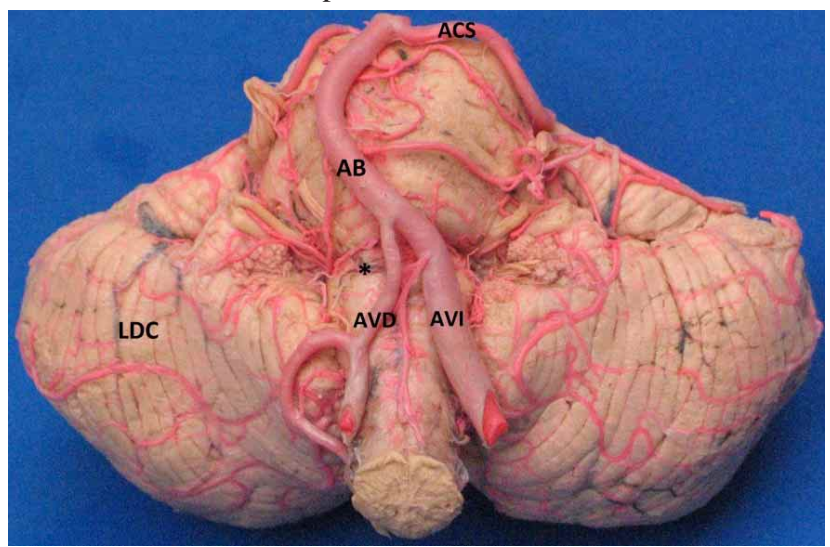

Fig. 4. Sistema arterial vertebrobasilar. Trayectoria sinuosa de la arteria vertebral. AB. Arteria basilar; LCD. Lóbulo derecho del cerebelo; AVD. Arteria vertebral derecha,hipoplásica; AVI. Arteria vertebral izquierda; ACS. Arteria cerebelar superior; Asterisco. Surco pontomedular.

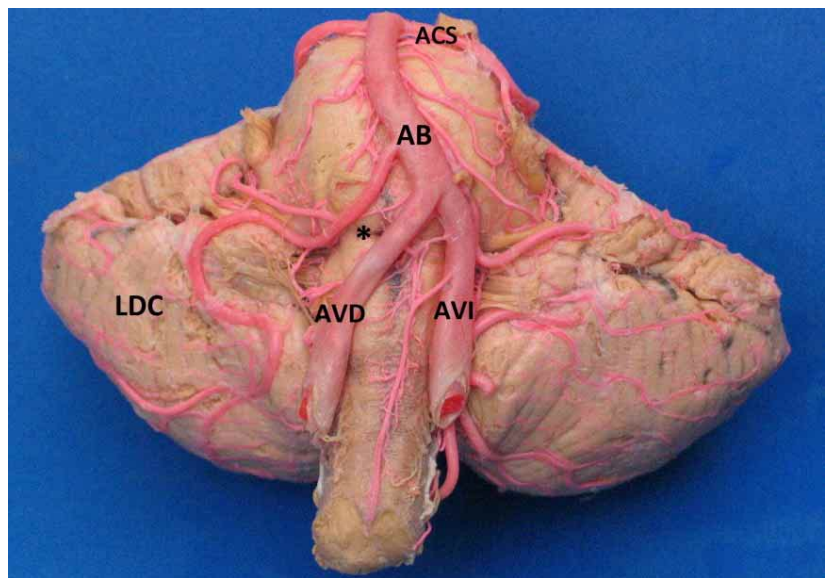

Fig. 5. Sistema arterial vertebrobasilar. Trayectoria desviada a la izquierda de la arteria basilar. AB. Arteria basilar; LCD. Lóbulo derecho del cerebelo; AVD. Arteria vertebral derecha; AVI. Arteria vertebral izquierda; ACS. Arteria cerebelar superior; Asterisco. Surco pontomedular. 


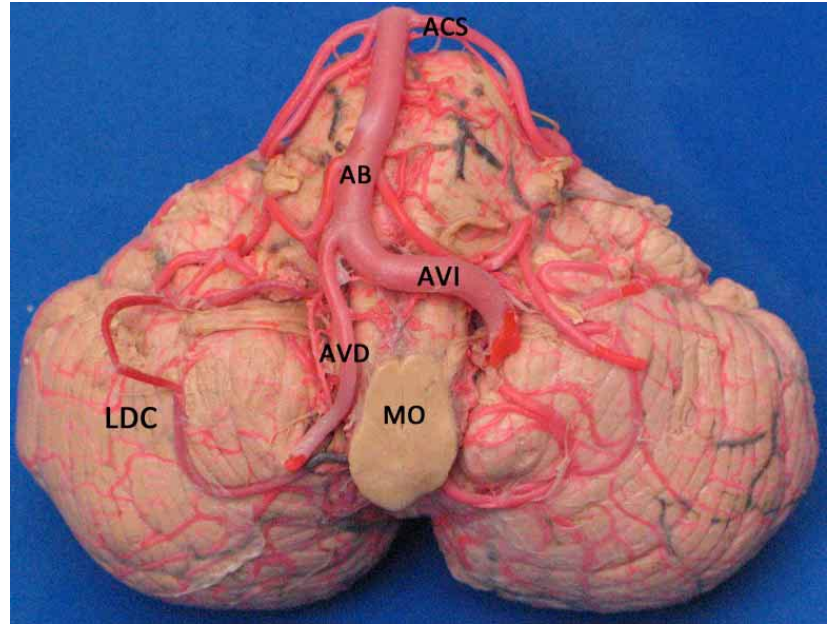

Fig. 6. Sistema arterial vertebrobasilar. Hipoplasia de la arteria vertebral derecha. AB. Arteria basilar; LCD. Lóbulo derecho del cerebelo; AVD. Arteria vertebral derecha que presenta marcada hipoplasia; AVI. Arteria vertebral izquierda; ACS. Arteria cerebelar superior; MD. Médula oblongada.

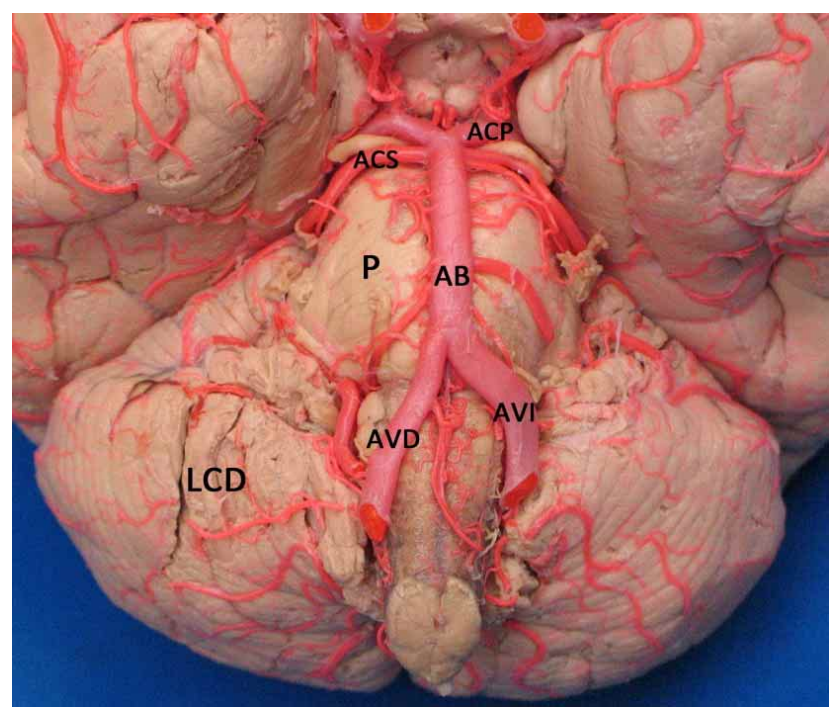

Fig. 6. Sistema arterial vertebrobasilar. Hipoplasia de la arteria vertebral derecha. AB. Arteria basilar; LCD. Lóbulo derecho del cerebelo; AVD. Arteria vertebral derecha que presenta marcada hipoplasia; AVI. Arteria vertebral izquierda; ACS. Arteria cerebelar superior; MD. Médula oblongada.

\section{DISCUSIÓN}

El surco pontomedular ha sido señalado por los textos clásicos de anatomía como el sitio en donde se unen los segmentos intracraneanos de las arterias vertebrales izquierda y derecha para dar origen a la $\mathrm{AB}$ (Afifi \& Bergman; Moore et al.; Drake et al.). Esta descripción puede considerarse como general en razón a que los diversos estudios sobre estas estructuras señalan que en menos de la mitad de las muestras evaluadas la unión vertebrobasilar se da al nivel del surco bulbomedular (Akar et al.; Santos-Franco et al.; Songur et al.; Stopford; Wojtowicz et al.). Nuestros hallazgos son concordantes con los reportes de Wojtowicz et al. y stopford (44-48\%). $\mathrm{El}$ origen de la $\mathrm{AB}$ proximal al surco pontomedular es descrito en la literatura con un rango del $12-53 \%$, tendencia que se mantiene con relación al origen distal al mencionado surco, el cual es reportado en un rango de $10,6-67 \%$ (Tabla I). La divergencia en las cifras reportadas por los diversos autores probablemente esté dada por múltiples factores como el tamaño de las muestras, metodología empleada y las características biológicas ancestrales que determinan la expresión variable de estas estructuras en los grupos poblacionales evaluados.

Las distancias promedio del origen de la $\mathrm{AB}$ proximal o distal al surco ponto medular, observadas en nuestra serie ( $4 \mathrm{~mm} ; 3,26 \mathrm{~mm}$ ) son similares a las reportadas por Pai et al. y Songur et al. Esta información es importante considerarla como referente en el manejo quirúrgico de procesos aneurismáticos, tumorales y ateromatosos ubicados en la fosa craneal posterior (Pai et al.; Santos-Franco et al. y Songur et al.; Pico et al.; Hong et al.).

La trayectoria rectilínea de la $\mathrm{AB}$ sobre la superficie del puente observada en nuestro estudio en el $80 \%$ de los sujetos jóvenes es concordante con los reportes de Schulte-Altedorneburg et al.; Wojtowicz et al.; Smoker et al. Cifras bajas de esta expresión, en un rango de 45$58,3 \%$ han sido reportadas en otros trabajos (Pai et al.; Santos-Franco et al. Torche et al. La desviación a derecha o izquierda de la $\mathrm{AB}$ ha sido señalada con una frecuencia del 35-52\% (Santos-Franco et al.; Torche et al.); mientras que los hallazgos observados en nuestra serie son similares a las incidencias reportadas en los trabajos de Schulte-Altedorneburg et al. y Wojtowicz et al. Igualmente, existe acuerdo en que la presentación sinuosa es la menos frecuente, en un rango de $1-10 \%$. Se destaca que Torche et al., reportan un $20 \%$ de AB sinuosas.

La asimetría de las arteria vertebrales, incluidas las hipoplasias y la presencia de la placas ateromatosas son factores que modifican las características del flujo sanguíneo de la $\mathrm{AB}$, lo cual determina que de manera paulatina el curso rectilíneo de esta arteria adopte una desviación a derecha e izquierda y en los casos severos llegue incluso a presentar una expresión sinuosa. Las diferencias etáreas de los especímenes evaluados en los diversos estudios explicarían en parte las divergencias observadas en los reportes de la trayectoria de la arteria basilar. 
La asimetría delas arterias vertebrales y especialmente su condición más severa, determina formas hipoplásicas que ocasionan además de alteraciones en la trayectoria de la $\mathrm{AB}$, trastornos en el flujo sanguíneo del tallo cerebral que pueden conducir a este nivel. La incidencia de hipoplasia de las arterias vertebrales en un rango de 2,4-12\% han sido reportados por Lovrencic-Huzjan et al., 1998; Perren et al., 2007); frecuencias moderadas entre las que se encuentra nuestro estudio en un rango de 15-. 26,5\% son reportadas por Park et al., 2007; Min et al., 2007; Chen et al., 2010; mientras que en estudios de sujetos con signos de infarto cerebral o neuropatía vestibular la hipoplasia de alguna de las arterias vertebrales es reportada en un rango de 42-54,8\% (Park et al.,; Chuang et al., 2011). La alta incidencia de hipoplasia de la $\mathrm{AV}$ en pacientes con infarto del tallo cerebral, especialmente al nivel del puente demuestra la correlación y significativo nivel de predicción para un evento isquémico probable en pacientes con esta condición anatómica (Chuang et al., 2009; Park et al.).

El menor calibre de la $\mathrm{AB}$ ha sido reportado en un rango de 3,1-3,3 mm (Hong et al.; Smoker et al.; Wollschlaeger et al.), en un rango medio (3,7-4 mm), en el que se ubica nuestro estudio, corresponden los reportes de Songur et al.; Santos-Franco y Wojtowicz et al. mientras que Pai et al. y Er et al. reportan los mayores calibres de la $\mathrm{AB}(4,3-4,8 \mathrm{~mm})$. Mandiola et al., 2004, reportan que el calibre de la $\mathrm{AB}$ se encontró en un rango de 4-5.9 $\mathrm{mm}$, en el $63.2 \%$ de sujetos Braquicéfalos y en el $62.5 \%$ del grupo dólico-mesocéfalico. Estas dimensiones no han sido mencionadas en otros estudios. Las diferencias en las técnicas empleadas, sitios de medición y tamaños de las muestras evaluadas puede explicar la considerable amplitud del rango que muestran los reportes de los calibres de esta estructura vascular.

En el presente estudio no se observó segmentos fenestrados en la $\mathrm{AB}$, aspecto que puede considerarse concordante con los trabajos previos que señalan la presencia de esta caracteristica entre el 0,6-1,5\% (Takahashi et al.,.,
1973; Sanders et al., 1993; Dodevski et al.; Goldstein et al.; Wollschlaeger et al.).Pueden considerarse a las fenestraciones como malformaciones en razón a que las modificaciones que generan en la dirección y fuerza del flujo sanguíneo puede alterar la resistencia de sus paredes y dar lugar a lesiones aneurismáticas (San-Galli et al. 1992, Sanders et al.).

La longitud de la $\mathrm{AB}$ encontrada en nuestra serie es ligeramente menor a la mayoría de reportes que señalan esta dimensión en un estrecho margen de 32,2 -33,3 mm(Akimoto et al.; Bush; Schulte-Altedorneburg et al.;Wojtowicz et al.; Wollschlaeger et al.). Mandiola et al., 1995, en su estudio con sujetos dolicomesocefálicos y braquio-cefálicos reporta la longitud de la $\mathrm{AB}$ en un rango de 31-37 $\mathrm{mm}$. Se destaca la menor longitud (24,9 $\mathrm{mm}$ ) reportada por Pai et al. Existe concordancia de la medición realizada en el presente trabajo, desde el origen de la AB hasta la emergencia de la arteria cerebelar superior $(28,1 \mathrm{~mm})$ con el reporte de Torche et al.

La bifurcación de la $\mathrm{AB}$ de manera aproximada se relaciona en los textos de anatomía con el surco superior del puente. Sin embargo, son pocos los estudios que matizan este aspecto de la $\mathrm{AB}$ y no existe acuerdo en torno a la estructura que permita referenciar la bifurcación de esta arteria. Pai et al., describen la división de la $\mathrm{AB}$ a 5,6 mm del cuerpo mamilar; Torche et al. reportan que el $92 \%$ de las arterias se dividen en la fosa interpeduncular; Mandiola et al., 1998 reportan dos tipos de división, alto o baja, con relación al surco superior del puente. En nuestra serie se discriminó el sitio de bifurcación al nivel del surco (55\%), por arriba o debajo de este surco de referencia.

El estudio de la expresión morfológica de la $\mathrm{AB}$ en sujetos colombianos provee información propia de referencia que puede ser contrastada con la obtenida en otros grupos poblacionales. Además, permite enriquecer el ejercicio de aplicaciones imagenológicas, el manejo de eventos clínicos y los abordajes quirúrgicos que comprometen al sistema vertebrobasilar.

Tabla I. Nivel de origen con relación al surco pontomedular de la arteria basilar en un muestra de 100 sujetos colombianos y en estudios previos. (Expresado en porcentajes)

\begin{tabular}{lcccccc}
\hline & $\begin{array}{c}\text { Stopford } \\
(\mathbf{1 9 1 6 )}\end{array}$ & $\begin{array}{c}\text { Wojtowicz } \\
\text { et al. } \mathbf{( 1 9 8 9 )}\end{array}$ & $\begin{array}{c}\text { Santos-Franco } \\
\text { et al. (2006) }\end{array}$ & $\begin{array}{c}\text { Akar et al } \\
(\mathbf{~ 1 9 9 4 )}\end{array}$ & $\begin{array}{c}\text { Songur et al. } \\
(\mathbf{2 0 0 8})\end{array}$ & $\begin{array}{c}\text { Presente } \\
\text { estudio }\end{array}$ \\
\hline Tamaño de muestra & 150 & 66 & 50 & 11 & 109 & 100 \\
Por encima del surco & 20 & 15,1 & 37 & 53 & 12,2 & 30 \\
Al nivel del surco & 48 & 44,4 & 27,5 & 36,4 & 20,8 & 43 \\
Por debajo del surco & 32 & 40,4 & 35,5 & 10,6 & 67 & 37 \\
\hline
\end{tabular}


FORERO, P. L.; BALLESTEROS, L. E \& RAMÍREZ, L. M. Morphological expression of the basilar artery. A study with Colombian population's autopsy material. Int. J. Morphol., 31(3):1090-1096, 2013.

SUMMARY: The basilar artery (BA) has a variable expression which is determinant in the etiology of clinical events that compromise the brain stem. The purpose of this study was to determine the morphological expression of BA in a Colombian population sample. We studied 100 brainstems of individuals which underwent an autopsy at the Instituto de Medicina Legal of Bucaramanga. The vertebra basilar system was perfused with synthetic resin and its calibers, paths, levels of origin and end of the BA were evaluated. The BA presented a rectilinear trajectory in $68 \%$, deviation to the right in $12 \%$, sinuous in $10 \%$ and deviation to the left in $10 \%$. The total length of the BA was $30.2 \mathrm{~mm} \mathrm{SD} \mathrm{4.07,} \mathrm{its} \mathrm{length} \mathrm{in} \mathrm{relation} \mathrm{to} \mathrm{the} \mathrm{origin} \mathrm{of} \mathrm{the} \mathrm{superior} \mathrm{cerebellar} \mathrm{artery} \mathrm{was} 28.1 \mathrm{~mm}$ SD 3.84 . The proximal and distal caliber of the BA were $3.96 \mathrm{~mm}$ SD $0.48 \mathrm{~mm}$ and $3.7 \mathrm{~mm}$ SD 0.58 respectively. In relation to the origin arteries of the $\mathrm{AB}$, hypoplasia was observed $(\geq 2 \mathrm{~mm})$ of the right vertebral artery in $8 \%$ and the left vertebral artery in $11 \%(\mathrm{P}=0.30)$. The origin of BA was observed at different spontomedullary groove levels: at level in $43 \%$, above it in $30 \%$ and below it in $27 \%$ of the cases. The sinuous and diverted paths from the BA observed and the presence of vertebral artery hypoplasia are slightly higher than those reported in previous studies, while its length is at medium range and its caliber is slightly lower than reported in the literature

KEY WORDS: Anatomical variation; Basilar artery; Vertebral artery; Brainstem.

\section{REFERENCIAS BIBLIOGRÁFICAS}

Afifi, A. \& Bergman, R. Functional neuro anatomy. 2nd ed. New York, MacGrawHill, 2008.

Akar, Z. C.; Dujovny, M.; Slavin, K. V.; Gomez-Tortosa, E. \& Aisman, J. I. Microsurgical anatomy of the intracranial part of the vertebral artery. Neurol. Res., 16(3):171-80,1994.

Akimoto, H. Roentgenological aspect of the basilar artery and its significance in clinical diagnosis - angiographical study a of the basilar artery. No Shinkei Geka, 7(12):1155-62,1979.

Bush, W. Beitrang zur Morphologie und Pathologie den Arteria Basilaris. Arch. Psychiatr. Neurkr., 208:326-44, 1966.

Chen, Y. Y.; Chao, A. C.; Hsu, H. Y.; Chung, C. P. \& Hu, H. H. Vertebral artery hypoplasia is associated with a decrease in net vertebral flow volume. Ultrasound Med. Biol., 36(1):3843, 2010.

Chuang, Y. M.; Chen, C. C. \& Lin, C. P. Vertebral artery hypoplasia may contribute to abnormal vestibular evoked myogenic potentials. Acta Neurol. Taiwan, 18(2):113-7, 2009.

Chuang, Y. M.; Chern, C. M.; Liao, W. H.; Hsu, L. C.; Lien C. F.; Lirng, J. F.; Shiao, A. S. \& Ko, J. S. Contribution of intracranial vertebral artery asymmetry to vestibular neuropathy. J. Neurol. Neurosurg. Psychiatry, 82(7):823-5, 2011.

Deng, D.; Cheng, F. B.; Zhang, Y.; Zhou, H. W.; Feng ,Y. \& Feng, J. C. Morphological analysis of the vertebral and basilar arteries in the Chinese population provides greater diagnostic accuracy of vertebrobasilar dolichoectasia and reveals gender differences. Surg. Radiol. Anat., 34(7):645-50, 2012.

Dimmick, S. J. \& Faulder, K. C. Normal variants of the cerebral circulation at multidetector CT angiography. Radiographics, 29(4):1027-43,2009.
Dodevski, A.; Lazareska, M.; Tosovska-Lazarova, D.; Zhivadinovik, J. \& Stojkoski, A. Basilar artery fenestration. Folia Morphol. (Warsz), 70(2):80-3, 2011.

Drake, R. L.; Vogl, A. W. \& Mitchell, A. W. Gray Anatomy for students. 2nd ed. Philadelphia, Churchill Livingstone, 2010.

Er, U.; Fraser, K. \& Lanzino, G. The anterior spinal artery origin: a microanatomical study. Spinal Cord., 46(1):45-9, 2008.

Goldstein, J. H.; Woodcock, R. M.; Do, H. M.; Phillips, C. D. \& Dion, J. E. Complete duplication or extreme fenestration of the basilar artery. AJNR Am. J. Neuroradiol., 20(1):149-50, 1999.

Hong, J. M.; Chung, C. S.; Bang, O. Y.; Yong, S. W.; Joo, I. S \& Huh, K. Vertebral artery dominance contributes to basilar artery curvature and peri-vertebro basilar junctional infarcts. $J$. Neurol. Neurosurg. Psychiatry, 80(10):1087-92, 2009.

Lovrencic-Huzjan, A.; Demarin, V.; Rundek, T. \& Vukovic, V. Role of vertebral artery hypoplasia in migraine. Cephalalgia, 18(10):684-6,1998.

Mandiola, E.; del Sol, M.; Olave, E.; Gabrielli, C. \& Prates, J. C. Consideraciones Anatómicas de la arteria basilar. Rev. Chil. Anat., 13(2):189-94, 1995.

Mandiola, E.; del Sol, M: Gabrielli, C.; Mizusaki, C. \& Prates, J. C. Bifurcation of the basilar artery and its relationship with the superior cerebellar artery at the proximal portion of the anterior pontine segment. Braz. J. Morphol. Sci., 15(1):29-33, 1998.

Mandiola, E.; del Sol M.; Olave, E.; Alarcón, E.; Montero, C.; Sanhueza, P. \& Oñate, J. C. Análisis biométrico entre mediciones bioantropológicas cefálicas y la arteria basilar. Int. J. Morphol., 22(2):165-8, 2004. 
Min, J. H. \& Lee, Y. S. Transcranial Doppler ultra sonographic evaluation of vertebral artery hypoplasia and aplasia. J. Neurol. Sci., 260(1-2):183-7, 2007.

Moore, K. L.; Dalley, A. \& Agur, A. M. Anatomía con orientación clínica. $6^{\mathrm{a}}$ ed. Buenos Aires, Lippincott Williams \& Wilkins, 2010 .

Pai, B. S.; Varma, R. G.; Kulkarni, R. N.; Nirmala, S.; Manjunath, L. C. \& Rakshith, S. Microsurgical anatomy of the posterior circulation. Neurol. India, 55(1):31-41, 2007.

Park, J. H.; Kim, J. M. \& Roh, J. K. Hypoplastic vertebral artery: frequency and associations with ischaemic stroke territory. $J$. Neurol. Neurosurg. Psychiatry, 78(9):954-8, 2007.

Perren, F.; Poglia, D.; Landis, T. \& Sztajzel, R. Vertebral artery hypoplasia: a predisposing factor for posterior circulation stroke? Neurology, 68(1):65-7, 2007.

Pico, F.; Labreuche, J.; Gourfinkel-An, I.; Amarenco, P. \& GENIC Investigators. Basilar Artery Diameter and 5-Year Mortality in Patients With Stroke. Stroke, 37(9):2342-7, 2006.

San-Galli, F.; Leman, C.; Kien, P.; Khazaal, J.; Phillips, S. D.\& Guérin, J. Cerebral arterial fenestrations associated with intracranial saccular aneurysms. Neurosurgery, 30(2):27983,1992 .

Sanders, W. P.; Sorek, P. A. \& Mehta, B. A. Fenestration of intracranial arteries with special attention to associated aneurysms and other anomalies. Am. J. Neuroradiol., 14(3):675-80,1993.

Santos-Franco, J. A.; de Oliveira, E.; Mercado, R.; Ortiz-Velazquez, R. I.; Revuelta-Gutierrez, R. \& Gomez-Llata, S. Microsurgical considerations of the anterior spinal and the anterior-ventral spinal arteries. Acta Neurochir. (Wien), 148(3):329-38, 2006.

Schulte-Altedorneburg, G.; Droste, D. W.; Popa, V.; Wohlgemuth, W. A.; Kellermann, M.; Nabavi, D. G.; Csiba, L. \& Ringelstein, E. B.Visualization of the Basilar Artery By Transcranial Color-Coded Duplex Sonography: Comparison With Postmortem Results. Stroke, 31(5):1123-7, 2000.

Smoker, W. R.; Price, M. J.; Keyes, W. D.; Corbett, J. J. \& Gentry, L. R. High-resolution computed tomography of the basilar artery: 1. Normal size and position. Am. J. Neuroradiol., 7(1):55-60,1986.

Songur, A.; Gonul, Y.; Ozen, O. A.; Kucuker, H.; Uzun, I.; Bas, O. \& Toktas, M. Variations in the intracranial vertebrobasilar system. Surg. Radiol. Anat., 30(3):257-64, 2008.

Stopford, J. S. The arteries of the pons and medulla oblongata. Part II. J. Anat. Physiol., 50(Pt. 3):255-80, 1916.

Takahashi, M.; Tamakawa, Y.; Kishikawa, T. \& Kowada, M. Fenestration of the basilar artery. Report of three cases and review of the literature. Radiology, 109(1):79-82, 1973.

Torche, M.; Mahmood , A.; Araujo, R.; Dujovny, M.; Dragovic, L. \& Ausman J. I. Microsurgical anatomy of the lower basilar artery. Neurol. Res., 14(3):259-62, 1992.

Uchino, A.; Saito, N.; Okada ,Y.; Kozawa, E.; Nishi ,N.; Mizukoshi, W.; Inoue, K.; Nakajima, R. \& Takahashi, M. Fenestrations of the intracranial vertebrobasilar system diagnosed by MR angiography. Neuroradiology, 54(5):445-50, 2012.

Wojtowicz, Z.; Zaluska, S.; Kis, G. \& Sawa, J. The basilar artery of humans. Ann. Univ. Mariae Curie Sklodowska. Med., 44:101-8,1989.

Wollschlaeger, G.; Wollschlaeger, P. B.; Lucas, F. V. \& Lopez, V. F. Experience and result with postmortem cerebral angiography performed as routine procedure of the autopsy. Am. J. Roentgenol. Radium Ther. Nucl. Med., 101(1):68-87, 1967.

Yoshimoto, H.; Maeda, H.; Aoyama, H.; Kanazawa, J.; Kitaoka, T. \& Uozumi, T. Enlargement of cerebellar arteriovenous malformation associated with fenestration of the vertebral artery: case report. Neurol. Med. Chir. (Tokyo), 32(8):5858,1992 .

Dirección para Correspondencia:

Dr. Pedro Luis Forero Porras

Cra. 32 \#29-31 Facultad de Salud

Universidad Industrial de Santander

Bucaramanga

COLOMBIA

Email: pedroluisforerop@ hotmail.com

Recibido : 17-10-2012

Aceptado: 27-04-2013 